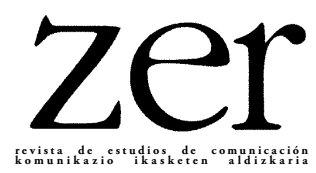

\title{
Semiótica de la intertemporalidad en medios videolúdicos. Propuesta de una matriz de análisis
}

\author{
Intenporalitatearen semiotika bitarteko bideo ludikoetan. \\ Analisi matrize baten proposamena \\ Semiotics of intertemporality in videoludic media. \\ Proposal of an analysis matrix
}

\author{
Cristian Felipe Parra $\star$ \\ Universidad Tecnológica de Chile INACAP
}

\begin{abstract}
RESUMEN: La mayoría de los videojuegos ludo-narrativos suponen relaciones temporales entre elementos narrativos de distinta naturaleza, principalmente elementos hipertextuales y elementos cibertextuales (narrativas autogeneradas por un simulador de realidad virtual). Estas relaciones tienen un ritmo que marca el desarrollo del discurso videolúdico en el universo narrativo. A partir de la identificación de los elementos constituyentes de ese ritmo intertemporal, este trabajo propone una tipología de relaciones que puede servir como matriz para el análisis del relato videolúdico en la medida que permite describir con mayor precisión la configuración temporal de este tipo particular de narrativas.
\end{abstract}

PALABRAS clave: Narración, hipertextualidad, games studies, videojuegos, semiótica, narrativa.

LABURPENA: Bideo-joko ludiko narratibo gehienek behin-behineko harremanak sortzen dituzte izaera ezberdineko elementu narratiboen artean, batez ere elementu hipertestualak eta elementu zibertestualak (errealitate birtualeko simulagailu batek automatikoki sortutako narratibak). Harreman horien erritmoak diskurtso bideo ludikoaren garapena zehazten du unibertso narratiboan. Erritmo intenportal hori osatzen duten elementuak identifikatzetik abiatuta, lan honen bidez, kontakizun bideo ludikoaren analisia egiteko matrize gisa balio dezakeen harremanen tipologia bat proposatzen da, narratiba mota partikular horren denbora konfigurazioa zehaztasun handiagoz deskribatzea ahalbidetzen duen neurrian.

HITZ-GAKOAK: Narrazioa, hipertestualitatea, games studies, bideo-jokoak, semiotika, narratiba. 
ABSTRACT: Most of ludo-narrative video games involve temporal relationships between narrative elements of different nature, mainly hypertextual and cybertextual elements (self-generated narratives by a virtual reality simulator). These relationships have a rhythm that marks the development of video videoludic discourse in the narrative universe. From the identification of the constituent elements of this intertemporal rhythm, the present work proposes a typology of relationships that can serve as a matrix for the analysis of the videoludic story insofar as it allows to describe with more precision the temporal configuration of this particular type of narratives.

KEYWORDS: Narrative, hyper-textuality, games studies, video games, communication, semiotics, narrativity.

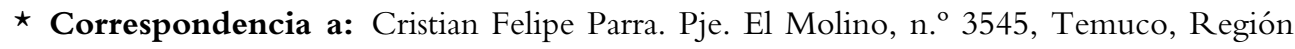
de La Araucanía, Chile. Código postal: 4781367. cristian.parra21@inacapmail.cl https://orcid.org/0000-0001-5954-2734

Cómo citar: Felipe Parra, Cristian. 2018. Semiótica de la intertemporalidad en medios videolúdicos. Propuesta de una matriz de análisis. Zer. 23(45). 57-74.

https://doi.org/10.1387/zer.19632

Recibido: 8 mayo 2018; aceptado: 15 octubre 2018

1137-1102 y 1989-631X / C 2018 UPV/EHU 


\section{Introducción}

Una de las principales formas de entretenimiento y uno de los fenómenos culturales más significativos de la modernidad es el videojuego. Esta evolución se refleja en el cada vez mayor tamaño de su industria. En 2014 este sector de la economía obtuvo ingresos por más de 75 mil millones de dólares y todo indica que esta industria seguirá cobrando importancia, no sólo en lo que se refiere al volumen de sus ingresos e inversiones, sino también en la progresiva complejidad formal de las obras del medio videolúdico tanto a nivel visual como argumental y técnico.

Este tipo de productos culturales han trascendido desde la clasificación de objetos tecnológicos o de ocio para convertirse en un actor relevante de la dinámica económica y socio-cultural. El desarrollo del videojuego aparece como un nuevo campo de creación, experimentación y expresión semiótica y ha inaugurado una ola de interés científico en torno a la descripción y comprensión de sus elementos constituyentes formales y su existencia en tanto forma de interacción simbólica y discursiva. Son estas problemáticas, entre otras, las que han logrado hacerse un espacio en el ambiente científico fundamentalmente a partir de la aparición de la revista de divulgación científica Games Studies, aparecida en el año 2001, que se dedicó a la investigación del juego en sus aspectos culturales estéticos y comunicativos.

En este contexto, el presente artículo revisa algunos de los avances teóricos más relevantes en torno al estudio de los videojuegos y presenta resultados de una investigación desarrollada recientemente (Parra, 2016) desarrollada con el propósito de aportar con herramientas metodológicas para el estudio de estos nuevos medios.

Las intuiciones teóricas y metodológicas para realizar esta aproximación analítica provienen de numerosos paradigmas disciplinarios que convergen en el estudio de estos fenómenos: semiótica, publicidad, psicología, psicoanálisis, marketing, educación, filosofia, etc. de todos ellos, la semiótica es tal vez una de las disciplinas que ha desarrollado de mejor manera las categorías de análisis para el abordaje de la narración en sus diversas formas.

En lo que sigue, presentaremos de manera general el marco conceptual del videojuego como fenómeno a la vez técnico y lúdico para luego introducir a la problemática del videojuego como unidad narrativa compleja.

\section{Antecedentes generales}

La realidad virtual (RV) se aborda habitualmente en términos de una particular colección de tecnologías («hardware») que involucran una pléyade de dispositivos que 
acaparan la atención del mundo actual (teléfonos y televisores inteligentes, computadores, dispositivos «wearables», etc). El foco parece estar puesto más sobre los avances electrónicos que sobre la experiencia que los grupos de sujetos experimentan en el uso de estos aparatos. No obstante, esta postura de orientación al artefacto electrónico adolece, desde el punto de vista de la comunicación, la semiótica y otras disciplinas afines, de una mirada que profundice en los procesos y efectos del uso de estos sistemas y sea en última instancia capaz de aportar lo que Steuer (1993) llamaría una estética desde la cual se pueda potenciar la creación de nuevos productos y un nuevo conjunto de métodos por los cuales los consumidores confien sus experiencias a estos medios de realidad virtual (RV).

Desde dicho punto de vista a la vez estético y funcional, la RV supone un intercambio de información, entre el medio y el sujeto, configurándose como vehículo para la materialización de un pensamiento lúdico y para la obtención de una experiencia emocional que es propuesta al jugador o actor quien al recibirla opera interpretativamente sobre ella decidiendo toda clase de acciones responsivas. Estas respuestas, en ocasiones, pueden escapar a la estructura prevista o bien, sencillamente seguir las alternativas prescritas como parte de la experiencia lúdica transmitida en el lenguaje que organiza el medio virtual. Estos sistemas de articulación de la información y las respuestas que obtiene, son claramente un fenómeno comunicacional toda vez que resulta de una mediación que tiene por objetivos lograr cierto tipo de respuesta a través de la disposición "estratégica» de significantes (discursos, signos, estímulos) presentados al lector. En términos de Umberto Eco (1981), el videojuego y la RV son fenómenos de cooperación textual, en tanto tales, deben ser entendidos como un artificio producido por uno o varios emisores, que solicita la participación activa de parte de un destinatario capaz de comprender sus diversos niveles de complejidad.

\subsection{LUdiCIDAD, NARRATIVA E HIPERFICCIÓN COLABORATIVA EN VIDEOJUEGOS}

Siguiendo los planteamientos de Aarseth (1997), toda investigación sobre el videojuego, desde cualquier perspectiva, se puede beneficiar en mayor o menor medida de un cierto conocimiento sobre las estructuras lúdicas y los procesos de experiencia de juego que subyacen a su diseño. En este sentido, gracias a los aportes cruciales de investigadores como Salen y Zimmerman (2004) y Juul (2005), se ha avanzado en el establecimiento de una teoría que se concentra en la composición formal del medio, dotada de un alto grado de abstracción.

No obstante lo anterior, el énfasis de la actual Ludología sobre los sistemas y desarrollo lúdicos del videojuego ha tendido a omitir la enorme importancia que un análisis centrado en los fenómenos y estructuras narrativas aporta a la comprensión cabal del fenómeno, si bien la discusión sobre la utilidad del enfoque narratoló- 
gico marcó los primeros años de los game studies y resulta en cierta medida obsoleta en este punto de su evolución, este tipo de análisis sigue planteando desafios descriptivo-analítico a la comunidad semiótica.

Las narraciones presentes en los videojuegos se realizan través de un dispositivo hipertextual cuyo origen se remonta a las formas vanguardistas de escritura y lectura que intentaron autores como Joyce o Cortázar mucho antes de que el hipertexto electrónico existiese como se conoce en la actualidad. Estas narraciones llevan al extremo también los postulados de la cooperación textual de Umberto Eco o de la Teoría de la recepción de Wolfgang Iser, en las cuales se concibe al lector en tanto sujeto activo y factor decisivo para la interpretación del texto. En el contexto videolúdico, el lector, al elegir sus propios trayectos de lectura «usurpa», en cierta forma, la función del autor tradicional a cuyo control parece escaparse el texto $(\mathrm{Pa}-$ jares, 1997).

El rol activo del destinatario en el contexto videolúdico queda de manifiesto también en la situación dinámica de coordinación entre hipertexto y cibertexto. Si bien el usuario tiene la posibilidad de seleccionar dentro de un abanico de líneas del guión, no interfiere mayormente sobre la configuración de la historia. No obstante, en los puntos intermedios de los itinerarios de lectura, es posible la acción «desregulada» de la acción de juego combinando estos itinerarios con las micronarraciones emergentes de la partida basadas en el comportamiento o decisiones particulares del jugador actual, es decir, explotando el cibertexto propio del medio videolúdico.

Dentro de este ambiente, en donde es posible relacionar ambos textos, entonces, existe una narrativa con cierto ritmo intertemporal. En un juego en que el ritmo lo impone el autor mediante una constricción temporal, el usuario o coautor, no tiene capacidad para cambiarlo, sino que tiene que adaptarse a él, pues de lo contrario no alcanzará sus objetivos. La constricción temporal del medio ludo-narrativo representaría, entonces, la puerta de entrada o el vector de unión entre las diferentes posibilidades de la experiencia de juego, esta es la relevancia de la constricción temporal del juego, por lo que identificar su organización constante en el medio contribuirá a poner en evidencia su valoración en términos de la estética del relato fantástico en ambientes de realidad virtual de naturaleza ludo-narrativo.

\section{Marco teórico}

El desarrollo actual de las tecnologías ha dado lugar casi irremediablemente al auge que vive actualmente el mercado de los videojuegos, es ligado a este auge que aparecen los denominados Games Studies (Máyrá, 2008), los cuales se definen como in- 
vestigaciones orientadas a manifestar las claves y las diversas implicaciones culturales — sociales, educativas o industriales - de este tipo de medios.

Desde una perspectiva epistemológica, los mencionados Game Studies siguen postulados de Kuhn (1978) o Popper (1974) en torno a las posibilidades y el interés científico desde la perspectiva que dicho interés debe ser común a un grupo de investigadores para que sus análisis y teorías cobren sentido y sus conclusiones sean relevantes para la comunidad científica. Sus teorías, de esta manera, se instalan tras los postulados de Kuhn (1987), es decir, consisten en un modo compartido por una colectividad de estudiosos que ven el fenómeno de interés bajo un mismo paradigma.

Así, esta corriente de estudios conforman una teoría como un acervo de supuestos en cierta medida establecida, evidente y vinculante que guía a determinado grupo de interesados en su búsqueda de explicar y comprender las implicancias del fenómeno del videojuego. Por lo tanto, sus teorías serán válidas en virtud de la aceptación que logren entre sus teóricos.

\subsection{El discurso VideOlúdico COMO JUEGO}

Si bien puede no existir precisión teórica para el análisis del texto lúdico, resulta evidente que el juego constituye un universo interpretativo de uso muy común en nuestra cultura conformando un medio que ha educado a todo tipo de personas prácticamente durante toda la existencia humana (Bettelheim, 1992). En esa línea, Huizinga (1998) pone de manifiesto una amplia variedad de casos que pueden conceptualizarse como juegos, poniendo de relieve la importancia y el interés del estudio de este tipo de manifestaciones culturales desde la Teoría del Texto. Este paradigma asienta un multiperspectivismo teórico que ayuda a analizar y comprender mejor el objeto en cuestión.

Dentro de esta diversidad de enfoques; la Antropología, las Matemáticas, la Psicología, la Teoría del Cibertexto y la moderna Ludología, permiten señalar cuatro ejes o relaciones semánticas esenciales, que conforman lo que Pérez (2010) denomina una estructura canónica del juego, a saber: «Sujeto-Entorno», «Medio-Experiencia», «Experiencia-Objeto»y «Redundancia-Variabilidad».

La primera relación («Sujeto-Entorno») se refiere a la cualidad que posee el juego en tanto a la obligatoriedad de tomar conciencia del resto de los sujetos que intervienen e integrarse en una organización ajena, es decir, esta tensión tiene reacción con la dialéctica «asimilación» «acomodación» de Piaget (2007) en su psicología del aprendizaje. 
Por su parte, la relación «Medio-Experiencia», distingue el sistema de la experiencia, es decir, contrasta el conjunto de reglas y el gameplay (las herramientas que posee el jugador para superar las dificultades que se plantean). Independiente de que cada juego tiene un «jugador modelo» (un jugador que sigue los itinerarios de acción previsto y sin demasiada flexibilidad en la ruta del héroe) existe una proyección simbólica que permite distinguir, por ejemplo, entre juegos deterministas o de superación.

La tercera relación «Experiencia-Objeto», enmarca al juego en lo que Egenfeldt-Nielsen (2008:37) denominan «experiencia diseñada con objetivo» y que son definidas desde la narrativa y la semiótica como relación «Sujeto-Objeto» (Bal, 1990). Se refieren los comportamientos aprendidos que sigue el jugador respecto de las situaciones de juego y sus actantes las que se interpretarán como «correctas» o «recomendables» en virtud de avanzar en su travesía, aventura o nivel.

Finalmente, el eje, «Redundancia-Variabilidad», se refiere a la clásica distinción propuesta por Caillois (2001) entre «ludus» y "paidea», es decir, entre formas altamente normadas de juego y la forma libre o sutilmente normada. Esta dicotomía va más allá de la definición de géneros de juego ya que todos ellos poseen alguna medida de Redundancia (acciones y eventos repetitivos) y un determinado nivel de Variabilidad (variantes o incluso aleatoriedad en algunos eventos de juego).

\subsection{El discurso VideOlúdico COMO UNIVERSO NARRATIVO}

Si la manifestación popular por antonomasia del cibertexto interactivo son los juegos y videojuegos, la principal manifestación popular del cibertexto no-interactivo son los mundos narrativos: mundos literarios de la ciencia-ficción, universos del cómic, etc. Aunque los videojuegos y este otro tipo de mundos narrativos no comparten la cualidad de la interactividad del usuario, sí comparten la cualidad de la «cibertextualidad» (Aarseth, 1997) o «textualidad ontológica» (McHale, 1987).

De esta manera, la cibertextualidad remite al estudio del texto con una dimensión sistémica indisociable del mismo, que subyace como entidad generativa de un espectro plural prácticamente innumerable de gameplays o narraciones posibles configurando un mundo ficciones que siguiendo a Dolezel (1999) resulta en una «macro-estructura generativa de historias».

En síntesis, el análisis del texto como mundo narrativo se orienta a las relaciones entre un individuo o colectivo protagonista y el entorno que le rodea (en sus dimensiones natural, cultural, social, etc.) diferenciándose del análisis del texto como narración que está centrado en las relaciones de tipo "sujeto-objeto» entre el «héroe» y sus objetivos (Pérez, 2010). 


\subsection{NARRACIONES PROTOTÍPICAS Y GAMEPLAY}

El análisis de la dimensión procesual del videojuego, entendido como universo narrativo, parte de la delimitación de un espectro amplio de patrones procesuales determinados a priori, en este caso "narraciones prototípicas» (Ruiz Collantes, 2009), macro-narraciones que resultan de la integración de varias narraciones con algún tipo de similitud sustancial.

El criterio fundamental para una concentración de narraciones particulares orientadas a la construcción de una composición textual prototípica unificadora es la identidad o similitud del objetivo principal en las diferentes narraciones. Sería posible considerar además como criterios relevantes la relación (identidad o similitud) entre el Sujeto protagonista de la acción y/o del Sujeto beneficiario de la misma en las narraciones. De esta manera, si se considera al juego como una composición textual englobada de varias narraciones particulares que se vinculan entre sí al compartir un objetivo a largo plazo, resultaría posible intercambiar el término de "narraciones» por "partidas» o "sesiones de juego» dejando a la vista la aplicabilidad de este enfoque teórico al análisis del videojuego toda vez que el jugador comprende la experiencia de juego a través de la sumatoria de diferentes sesiones de interactividad (partidas) que le permite avanzar hacia el logro del objeto final del juego.

Según este enfoque teórico, la lectura del gameplay de un videojuego puede dar lugar a una única narración prototípica o a muchas. En los videojuegos con gameplay «rígida», una sola narración prototípica podría condensar toda la experiencia narrativa mientras que, en los videojuegos con gameplay más abierta, se debería definir un espectro indeterminado de «narraciones prototípicas», que responderán, cada una, a una experiencia de juego prototípica respecto a distintos objetivos o estados finales particulares del videojuego (Pérez, 2014).

La afinidad entre los conceptos de narración prototípica y gameplay (como experiencia de juego prototípica vinculada a un objetivo determinado), invita a considerar un estudio de la dimensión procesual del texto videolúdico donde se complementen el análisis de la interactividad y formas lúdicas con técnicas e instrumentos del análisis narrativo.

\subsection{LA GAMEPLAY COMO ESTRUCTURA NARRATIVA TRANSFORMACIONAL}

El modelo transformacional de la narrativa es, a diferencia de otros como el actancial o dramático, un modelo concebido en clave dinámica o diacrónica, con acento temporal. Desde esta mirada, la estructura mínima de la narratividad se basa en el encadenamiento de "enunciados de estado» y «enunciados de acción»: mientras los enunciados de estado son, esquemáticamente, proposiciones de "poseer / no poseer» un 
objeto ( $\mathrm{S} \cap \mathrm{O} / \mathrm{SUO}$ ), los enunciados de acción son, esquemáticamente, proposiciones de «hacer poseer / hacer no poseer» un objeto (SUO $\mathrm{S} \cap \mathrm{O} / \mathrm{S} \cap \mathrm{O} \mathrm{SUO}$ ).

De esta forma, un relato mínimo se puede definir como un cambio de estado con acciones/transformaciones intermedias. Tomando un ejemplo de Courtés (1980: 107-136), la expresión mínima del cuento de la Cenicienta consistiría en un cambio de estado entre las situaciones de «Príncipe y Cenicienta separados» y «Príncipe y Cenicienta juntos», a través de la función de transformación (Ft): "casarse».

Esta unidad mínima del relato constituye el núcleo de un programa narrativo, y por tanto podría desplegarse en una estructura actancial, pero si se escoge el modelo transformacional lo importante no es tanto observar las particularidades actanciales de cada programa y subprograma narrativo sino atender al encadenamiento en el tiempo de sucesivos programas narrativos.

Al final, esto ofrece algo así como una representación «heurística» de la estructura narrativa, una representación de las acciones sucesivas necesarias para resolver un problema (conseguir un objetivo).

\subsection{Temporalidad VIDEOLÚdicA y ORDEN}

La temporalidad y orden del argumento videolúdico de la historia puede ser de dos tipos, dando saltos atrás en el tiempo o «flashbacks» (Analepsis) o bien saltos adelante en el tiempo o «flashforwards» (Prolepsis) (Genette, 1987). La relación historia-argumento de un videojuego puede contener los mismos tipos de saltos temporales que una novela o una película, pero en el caso de los videojuegos es relevante contrastar si la interacción del jugador resulta decisiva o no dentro de uno de estos saltos.

La duración temporal de la narración consiste en la tensión manipulada (constricción temporal) de la duración de determinados pasajes de la historia a nivel argumental. En función de la relación entre la duración de tiempo en la historia (TH) y en el argumento (TA). Genette (1987) distingue cinco tipos de manipulaciones de la duración temporal en la narración:

- Adecuación: $\mathrm{TA}=\mathrm{TH}$

- Resumen: TA $<$ TH

- Dilatación: TA $>$ TH

- Elipsis: TA $<<\mathrm{TH}$

- Pausa: TA $>>$ TH

Queda de manifiesto que tal como una película (argumento) de 90 minutos puede contener todas las peripecias de una travesía de 100 días (historia), si se pone en rela- 
ción la duración temporal narrativa con el «ritmo» temporal del juego, surgen algunas variaciones expresivas del medio, surgiendo múltiples combinaciones de manipulaciones de temporalidad narrativas y manipulaciones de temporalidad lúdicas con diverso valor interpretativo en la estructura de videojuegos.

\section{Categorías fundamentales del medio ludo-narrativo}

Este estudio busca la descripción del funcionamiento de los mundos narrativos en torno al ritmo intertemporal o estructuras de variabilidad que dan espacio para experimentar las condiciones del medio generativo. En esencia, los arquetipos teóricos que se desarrollan aquí son susceptibles de ser aplicados a cualquier tipo de videojuego, aunque naturalmente resulten en especial apropiados para el estudio de videojuegos explícitamente narrativos (videojuegos de rol, aventuras gráficas, etc.).

A partir de los paradigmas estudiados se elaboró una matriz de análisis que operacionaliza las categorías y dimensiones identificadas en juegos previamente caracterizados. Estos elementos configuran la historia videolúdica del juego. Lo que se buscó fue reconocer las figuras (actantes), sus programas narrativos a nivel genérico y su incidencia o valor a nivel cibertextual. Este procedimiento está orientado a identificar los elementos constituyentes de naturaleza ludo-narrativas presentes en videojuegos relevantes, como primer objetivo.

Mediante esta categorización, los elementos encontrados anteriormente establecen si las unidades de análisis tienen una incidencia directa o indirecta en el relato estableciendo su valor temático, es decir, determinar si corresponden al núcleo del relato o a satélites dentro de él, logrando establecer los elementos constituyentes ludo-narrativos en relación a su acción o rol en el relato del juego.

Estas categorías constituyen los elementos más signifcativos que cruzan al videojuego en su dimensión narrativa hipertextual y lúdica generativa (cibertextual) y se han obtenido del estudio del marco teórico desarrollado.

\subsection{Categoría I. Acciones Significativas}

La primera categoría caracterizada implica las distintas posibilidades derivadas de las respuestas del jugador a las complejidades que le presenta el sistema las cuales modelan el universo ficticio en el marco de los límites previstos por el diseñador del juego. Se configura así una forma de narratividad lúdica definida por lo posible y lo necesario que distingue entre núcleo y satélites. La observación de este modo de operar en el relato del juego, permitirá identificar la emergencia de unidades de narración y con ello, la cristalización de un interespacio de libertad para el usuario. 
Una acción significativa es el producto de un tipo de relación en un contexto de oposición que encuentra el jugador o usuario dentro del campo del medio lúdico, este posicionamiento implica ciertas relaciones dentro del circulo mágico y se constituyen como dimensiones de esta categoría:

Relación Sujeto/entorno: Implica la cristalización del Sujeto/jugador como entidad semiótica mediante el diseño de reglas que modela competencias de actuación (Greimas y Courtés) y procesos transformacionales de acción-efecto (Salen y Zimmerman). Estos procesos toman cuerpo mediante la puesta en escena del entorno del juego compuesto de actores opositores y todo tipo de actante semiótico cuyas propiedades y relaciones particulares permiten la proyección del texto como mundo. De esta manera, la dimensión operacionalmente implicará la acomodación del sujeto y la temporalidad significativa en función de la relación entre la duración de tiempo en la historia (TH) y en el argumento (TA). Hay cinco tipos de manipulaciones de la duración temporal en la narración:

Escena $(\mathrm{TA}=\mathrm{TH})$ : Genette $(1987)$ en torno a los tiempos narrativos el tiempo del relato (tiempo de argumento para estos efectos) presenta un caso de isocronía en el que se supone que la duración diegética es idéntica a la duración narrativa. La narración en curso se extiende, en la diégesis, en la misma extención que el tiempo indispensable para narrarlo. Aquí la relación entre el tiempo argumento y tiempo historia es igual. El desarrollo del argumento presentado es significativo para el relato núcleo del universo narrativo.

Sumario $(\mathrm{TA}<\mathrm{TH})$ : Según Genette, se utiliza para resumir un tiempo diegético que se supone más largo y se lee: el Tiempo del Argumento es más corto que el Tiempo de la Historia. El énfasis es mayor hacia el tiempo historia, se presentan acciones tendientes a reforzar la historia ya sea mediante PNJs o secuencias animadas y no mediante acciones que deba desarrollar el jugador.

Dilatación (TA > TH): En un texto narrativo de segmentos descriptivos o comentativos su efecto consiste en alargar indefinidamente el tiempo del relato. Este procedimiento se corresponde, aunque en sentido inverso, al del resumen. Se lee Tiempo del Argumento es más importante que el Tiempo de la Historia. Corresponde a argumentos que si bien se alejan del eje de lectura lineal de la historia, proporciona recursos necesarios para completarla.

Elipsis (TA $<<\mathrm{TH}$ ) Corresponde a un silencio o recorte (narrativo) acerca de ciertos acontecimientos que, según la diégesis, han tenido lugar.

Pausa (TA $>>$ TH) Este caso corresponde a que una duración determinada del relato no le corresponde ninguna duración diegética (de la historia) con lo cual el 
Tiempo del Argumento es infinitamente más importante que el Tiempo de la Historia, dado que no tiene ninguna relación con ella.

Relación Medio/Experiencia: Corresponde a la relación generativa entre el «sistema de juego» incentivos que contienen un comportamiento previsto del jugador, en tanto que «jugador modelo» (Frasca, 2001) y la "experiencia de juego» (gameplay). Discursivamente, el sistema de juego se configura como un Medio generativo de experiencias suyo alcance simbólico permite distinguir, por ejemplo, entre juegos «de superación» en oposición a juegos «deterministas». Se identificarán acciones deterministas hacia un jugador "modelo» o de «liberación» dependiendo de la obligatoriedad del jugador a construirse o no de una forma determinada por el medio.

Relación Experiencia/Objeto: En relación con el Objetivo final o parciales en el juego, la gameplay constituye al mismo tiempo una "experiencia prescrita» lo que quiere decir que los comportamientos aprendidos que sigue el jugador respecto de las situaciones de juego y sus actantes podrán ser interpretadas como «correctas» o «recomendables» en virtud de avanzar en su travesía, aventura o nivel. Estas formas de interacción que se aprenden respecto a un objetivo dado forman parte del núcleo de significación del discurso lúdico en su forma de prescriptor de rutinas de resolución de problemas. De esta manera, la interpretación de la gameplay de un videojuego puede permitir identificar una única narración prototípica o muchas, según el caso. En los videojuegos con gameplay «rígida», una sola narración prototípica condensaría toda la experiencia narrativa que ofrece el videojuego en lo fundamental mientras que, en los videojuegos con gameplay más abierta, se debería definir un espectro amplio de «narraciones prototípicas», que responderán, cada una, a una experiencia de juego prototípica respecto a distintos objetivos o estados finales particulares del videojuego.

Relación Redundancia/ Variabilidad: Representación temática que devela la metáfora de actante. Esta dimensión implica los dos grandes géneros de juego propuestos por Caillois en su idea de sociología del jugar: 1. Paidia: corresponde a un valor de alegría o «disfrute» de la experiencia del acto del juego. Esta categoría, según la propuesta teorética de Caillois, representa un alto valor a la libertad del jugador por sobre las normas del juego; 2. Ludus, esta dimensión se corresponde en Caillois con la estructura formal basado en reglas y el comportamiento del juego de acuerdo a la estructura lineal del autor extradiegético, su exacerbación se correspondería con un bajo nivel hiperficticio.

\subsection{Categoría II: Programas narrativos}

Asociado a su esfera de acción, el sujeto será visto desde el modelo transformacional de la narrativa. Desde esta perspectiva, la estructura mínima de la narratividad se basa 
en el encadenamiento de «enunciados de estado» y «enunciados de acción»: mientras los primeros son, esquemáticamente, proposiciones de «poseer / no poseer» un objeto ( $\mathrm{S} \cap \mathrm{O} / \mathrm{SUO}$ ), los enunciados de acción son, esquemáticamente, proposiciones

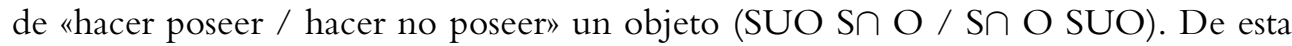
forma, un relato mínimo se puede definir como un cambio de estado con acciones/ transformaciones intermedias (Parra, 2014). Esta unidad mínima del relato constituye el núcleo de un programa narrativo, y por tanto podría desplegarse en una estructura actancial, pero si se escoge el modelo transformacional lo importante no es tanto observar las particularidades actanciales de cada programa y subprograma narrativo sino atender al encadenamiento en el tiempo de sucesivos programas. Desde esta perspectiva, un cambio de estado en la narración entraña otro de nivel inferior, y así sucesivamente (Parra, 2014).

\subsection{Categoría III: Valor intertemporal}

El valor intertemporal identifica la tensión entre el usuario y el autor por tomar desiciones generativas del argumento cibertextual en el recorrido adaptativo que realiza el jugador por el medio, en este sentido, se busca discriminar entre los enunciados de estado y de acción (que tienen una segunda categorización de acuerdo a su relación medio/experiencia) que se condicen o no con una libertad o paidia significativa en el medio lúdico logrando identificar la constricción temporal que supone la relación emergente entre el sujeto y entorno la cual puede ser positiva (no representa una constricción temporal) o negativa (representa una constricción temporal).

Considerando todo lo anterior es que se puede hacer una relación directa entre las categorías y el objeto de estudio de manera tal de poder operacionalizar cada una de éstas. En este sentido, es que se analizaron las categorías (y las dimensiones obtenidas de cada una), de modo de apreciar su incidencia relativa de acuerdo a los objetivos planteados.

Luego de practicar dicho análisis se procedió a indicar la incidencia relativa de cada dimensión tomando como referencia la observación del comportamiento y los roles presentes de acuerdo a los criterios declarados referidos a cada dimensión, por lo que se usa como criterio de medición la clasificación de los personajes y su valor en referencia en el análisis a cada dimensión, de modo de poder tener una apreciación de cuales dimensiones se reiteran consistentemente en los juegos.

De esta forma, las categorías "Acción significativa» y "Programa narrativo» son identificadas entre los actantes que se presentan ante el avatar del jugador, mientras que los constituyentes de la categoría «Valor intertemporal» son calificados de acuerdo a un criterio de exclusión expresado en los términos de "positivo" y «negativo». 
Finalmente se construyó una tabla referencial resumen, donde se identifica y cuantifican los actantes y su clasificación relativa de cada dimensión analizada en relación en su presencia por nivel del jugador basándose en el Marco Teórico en el criterio del investigador, resultando una tabla como la siguiente:

\begin{tabular}{|c|c|c|c|c|c|}
\hline \multirow{2}{*}{$\begin{array}{l}\text { Unidades } \\
\text { componentes } \\
\text { del nivel } \\
\text { macro- } \\
\text { estructural }\end{array}$} & \multicolumn{3}{|c|}{ Acciones significativas } & \multirow{2}{*}{$\begin{array}{l}\text { Programas } \\
\text { Narrativos }\end{array}$} & \multirow{2}{*}{$\begin{array}{l}\text { Valor } \\
\text { intertemporal }\end{array}$} \\
\hline & Sujeto/entorno & $\begin{array}{l}\text { Medio/ } \\
\text { experiencia }\end{array}$ & $\begin{array}{l}\text { Redundancia/ } \\
\text { Variabilidad }\end{array}$ & & \\
\hline $\begin{array}{l}\text { Corresponde a la } \\
\text { identificación del } \\
\text { fragmento del } \\
\text { relato que } \\
\text { detona la acción } \\
\text { del jugador, } \\
\text { corresponderia a } \\
\text { las misiones o } \\
\text { quest }\end{array}$ & $\begin{array}{l}\text { Clasifica la } \\
\text { narración puesta } \\
\text { en escena y que } \\
\text { concreta como } \\
\text { entidad semiótica } \\
\text { al jugador } \\
\text { involucrando al } \\
\text { entorno del } \\
\text { juego. La } \\
\text { dimensión } \\
\text { operacionalmente } \\
\text { implicará la } \\
\text { acomodación del } \\
\text { sujeto y la } \\
\text { temporalidad } \\
\text { significativa, en } \\
\text { función de la } \\
\text { relación entre la } \\
\text { duración de } \\
\text { tiempo en la } \\
\text { historia (TH) y } \\
\text { en el argumento } \\
\text { (TA) }\end{array}$ & $\begin{array}{l}\text { Se identificarán } \\
\text { acciones } \\
\text { deterministas, } \\
\text { hacia un jugador } \\
\text { "modelo" o de } \\
\text { "liberación" } \\
\text { dependiendo de } \\
\text { la obligatoriedad } \\
\text { en las acciones } \\
\text { del jugadores } \\
\text { virtud a } \\
\text { construirse o no } \\
\text { de una forma } \\
\text { determinada } \\
\text { dentro del juego. }\end{array}$ & $\begin{array}{l}\text { Identifica el } \\
\text { acento } \\
\text { argumental de los } \\
\text { dos grandes } \\
\text { géneros de juego } \\
\text { propuestos por } \\
\text { Caillois en su } \\
\text { idea de sociología } \\
\text { del jugar } \\
\text { "Ludus"| } \\
\text { "Paidea". }\end{array}$ & 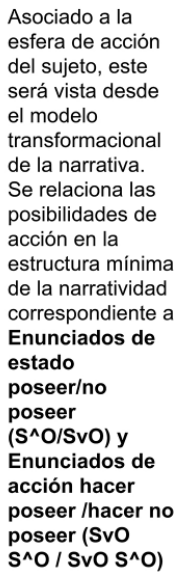 & $\begin{array}{l}\text { Identifica la tensión } \\
\text { entre el usuario y el } \\
\text { autor por tomar } \\
\text { desiciones } \\
\text { generativas del } \\
\text { argumento } \\
\text { cibertextual. La } \\
\text { constricción } \\
\text { temporal que } \\
\text { supone la relación } \\
\text { emergente entre } \\
\text { el sujeto y } \\
\text { entorno puede ser } \\
\text { positiva (no } \\
\text { representa una } \\
\text { constricción } \\
\text { temporal) o } \\
\text { negativa } \\
\text { (representa una } \\
\text { constricción } \\
\text { temporal). }\end{array}$ \\
\hline
\end{tabular}

En esta matriz se observan las categorías y dimensiones que daría cuerpo al relato videolúdico, su uso está destinado a aplicar a videojuegos que, en todo caso, deben cumplir cuatro características fundamentales que permiten aplicarla y obtener infromación relevante en torno a la presencia de constricciones temporales y ritmo intertemporal.

\section{Consideraciones finales}

El instrumento de análisis presentado ofrece en sí mismo una amplia gama de aplicaciones al universo semiótico donde se enmarca. La caracterización de elementos constituyentes del relato videolúdico y su sistematización en una matriz de análisis ludo-narrativa permite observar la existencia de acciones significativas, programas narrativos y constricciones temporales que se organizan de acuerdo a los postulados clásicos en torno al relato - usualmente en forma de argumento hipertextual linealramificado- A su vez, el ordenamiento de categorías ofrecido sistematiza estos ele- 
mentos constituyentes del relato videolúdico permitiendo no sólo una aplicación dentro de los parámetros de la matriz sino también de manera modular — análisis de las categorías y sus dimensiones separadamente de la matriz de intertemporalidadlo que amplía su potencialidad como herramienta en otras áreas fuera del ambiente estrictamente narrativo.

De esta manera, por ejemplo, un análisis de acciones significativas ofrece la posibilidad de estudio a nivel de las relaciones entre la acomodación, tiempo y tensión Ludus/Pidea; el análisis de programas narrativos, ofrece una visión clásica de de la estructura del discurso - o relato - en términos de enunciados de estado; mientras que el reconocimiento del valor intertemporal permite visualizar la estructura rítmica de la constricción temporal de un medio dado. En suma, si bien se provee de un instrumento teórico que permite el análisis global del tiempo, tensión y narración ludonarrativa en un ambiente de cibertextualidad, dicha herramienta alcanza un valor didáctico en el estudio de los videojuegos y útil en otras áreas con intereses más específicos.

En lo referente a la futura aplicación de esta matriz, se hace necesario establecer algunas precisiones sobre las características que debe cumplir una muestra o videojuego susceptible de analizar bajo la mirada aquí planteada, esto debido a que existe tal cantidad de variaciones entre los videojuegos que la tarea de clasificarlos ha resultado un ejercicio teórico complejo para los investigadores que han incursionado en estas tareas. Lo anterior hace evidente considerar que para analizar estructuras internas de los juegos deberá tomarse en alta consideración que las muestras contemplen las suficientes similitudes para compararlas y diferencias para acotarlas coherentemente y que hagan comprensible la aplicación de una misma matriz de análisis. Dificilmente podría aplicarse el mismo instrumento a juegos con diferentes énfasis de categorización (un juego orientado a una curva de dificultad, como Tetris, o uno orientado a una curva dramática como Assassin's Creed) y esperar resultados concluyentes o verosímiles.

\subsection{Criterios de SElección para una muestra o APLiCACión}

Por otro lado, se ha observado que hay claras diferencias entre los tipos de clasificación que provee la literatura especializada, aunque para efectos de la investigación en curso deberán tomarse desiciones sobre las pautas de clasificación considerando los intereses particulares aquí planteados. En el comienzo del presente documento se señalan los puntos comunes entre las teorías del hipertexto y de cibertexto llegando a señalar que el análisis será sobre juegos ludo-narrativos (definidos más adelante como universos narrativos), por tanto cabrá señalar las condiciones que deberá cumplir un juego de esa naturaleza las cuales se desprendan como criterio general de una "presencia de historia». 
Si se concibe al jugador sólo como «(co)autor» del videojuego y en ningún grado como «actor» dentro del mismo juego, entonces se dirá que todo videojuego contiene infinitas historias posibles. Por el contrario, si se considera al jugador sólo como «actor» dentro de un juego y en ningún grado como «(co)autor», entonces se dirá que un videojuego tiene una historia o una serie de historias fijas, sobre las que la intervención del jugador no tiene nunca importancia alguna.

Independientemente del rol que desempeñe el jugador, el primer rasgo para el análisis considerará el sólo hecho de existir una historia o relato que guíe el juego a un nivel profundo y no meramente contextual, identificar una historia involucra:

I. La posibilidad de segmentar globalmente la linealidad temporal de la narración/gameplay identificando unidades y acontecimientos fundamentales. Esto se refiere a la existencia de unidades componentes del nivel macroestructural que se correspondan a los tres actos canónicos de la narrativa o a una arquitectura de episodio.

II. Definir/interpretar el contenido de dichas unidades esenciales con un alto grado de generalización/abstracción. Detalles que caracterizan a un existente o evento de la narración (p. ej., traje gris, cicatriz en mejilla, sombrero de fieltro, pistola oculta) tienden a ser reducidos en el nivel de la «historia» a nociones más simples que incluyan todos los detalles posibles (p. ej., "gángster»).

III. Organizarse conforme a un orden cronológico factual y fijo/invariante. Se debe considerar que los episodios esenciales, en tanto que unidades de la «historia», deben estar organizados según un orden fijo y linealizado.

IV. Existir «acciones narrativamente esenciales» del jugador dentro del juego. Es decir, que existan acciones del jugador que afectan al desarrollo de acontecimientos esenciales de la narración (nivel profundo).

Finalmente, para la correcta aplicación de la matriz de análisis, deberá existir un argumento hipertextual que permita distinguir entre estructuras hipertextuales que sostienen un orden lineal fijo de los nodos argumentales (escenas, misiones, acontecimientos relevantes de la trama), es decir "vector de ramas laterales» y estructuras hipertextuales que presentan variabilidad en la ordenación lineal de los nodos argumentales, o sea, estructuras argumentales en forma de red. Este punto garantizará estar en presencia de un videojuego con historia y relato hipertextual, sin embargo, la distinción entre las estructuras prototipicas queda fuera como criterio de clasificación, es decir, sólo tendrá valor en tanto estructura hipertextual. 


\section{Referencias}

Aarseth, E. (1997) Cybertext: perspectives on ergodic literature. Baltimore: Johns Hopkins University Press.

AEVI, Asociación Española de Videojuego (2015) El consumo global de videojuegos en España superó los 1.000 millones de euros en 2015. Recuperado de:http://www.aevi. org.es/consumo-global-videojuegos-espana-supero-los-1-000-millones-euros-2015/

Bettetini, G. (1996) L'audiovisivo. Dat cinema ai nuovi media. Milan: Bompiani.

Biocca, F., \& Delaney, B. (1995). Immersive virtual reality technology. En F. Biocca \& M.R. Levy (Eds.), Communication in the age of virtual reality (pp. 57-124). Hillsdale, NJ: Lawrence Erlbaum Associates.

Colombo, F. Y Eugeni, R. (1996) II testo visibile. Roma. Editori Riuniti.

Genette, G. (1987) Figuras III. Barcelona: Editorial Lumen.

Eco, U. (1979) Lector in fabula. La cooperazione interpretativa nei testi narrativi- Lector in fabula. La cooperación interpretativa en el texto narrativo, trd.: Ricardo Pochtar; Lumen, Barcelona, 1981.

Eco, U. (1990) I limiti dell'interpretazione. Milan: Bompiani.

Eco, U. (1994) Sei passeggiate nei boschi narrativi. Milan: Bompiani.

Frasca, G. (2001) Videogames of the oppressed: videogames as a means for critical thinking and debate. Tesis del Master of Information Design and Technology. Georgia Institute of Technology. Consultado de: http://www.ludology.org/articles/thesis/ [06/12/2016].

Juul, J. (2005) Half-Real: videogames between real rules and fictional worlds. Cambridge: MIT Press.

Landow, G. (2009) Hypertext 3.0. Barcelona: Paidós.

Navarrete, Gómez, Pérez, J. (2014). Una aproximación a los paradigmas de la Teoría del Videojuego. Retrieved from https://idus.us.es/xmlui/bitstream/handle/11441/24726/ zer37-06-navarrete.pdf?sequence $=1$

Nelson, T. (1992) Literary Machines 93.1. Sausalito: Mindful Press.

Pajares, S. Las posibilidades de la narrativa hipertextual, Obtenida el 09 de agosto de 2015. Consultado de: http://pendientedemigracion.ucm.es/info/especulo/numero6/s_ pajare.htm [12/01/2017].

Parra, C. (2016). Ritmo intertemporal y estética del relato en medios ludo-narrativos. Configuración de la historia y el argumento videolúdico (tesis de maestría). Universidad de la Frontera, Temuco, Chile.

Pérez, Ó. (2014, October 2). El significado en juego: sobre la dimensión lúdica del discurso del videojuego. Retrieved from http://ruc.udc.es/dspace/bitstream/ handle/2183/13489/CC-130_art_185.pdf?sequence=1

Salen, K. Y Zimmerman, E. (2004) Rules of Play: game design fundamentals. Cambridge, Londres: MIT Press. 
Schroeder, R. (1996) Possible worlds: The social dynamic of virtual reality technology. Boulder. Westview Press.

Scolari, C. (2003) Hipertextos, Interfaces, Interacciones. de Signis n. ${ }^{\circ}$ 5. Editorial Gedisa, Barcelona. PP 23-26.

Steuer J.S. (1992) Defining virtual reality: Dimensions determining telepresence, Journal of Communication n. ${ }^{\circ} 42$, pp. 73-93. Consultado de: http://www.cybertherapy.info/ pages/telepresence.pdf [06/12/2016]. 